\title{
QUANTIFYING THE PHYSICAL DEMANDS OF SMALL SIDED GAMES IN RUGBY UNION: CONTACT VS. NON-CONTACT
}

\author{
Lydia Chadwick, ${ }^{A, B, C, D}$ Richard M. Page, ${ }^{A, D}$ Ben Langley, ${ }^{A, C, D}$ \\ Sport and Physical Activity, Edge Hill University, Ormskirk, Lancashire, UK

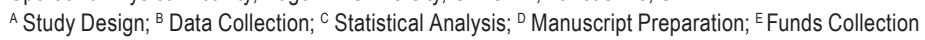 \\ Address for corpespondence: \\ Ben Langley \\ Sport and Physical Activity, Faculty of Arts and Sciences, Edge Hill University \\ St Helens Road, Ormskirk, Lancashire, L39 4QP, UK \\ E-mail: Ben.Langley@edgehill.ac.uk
}

\begin{abstract}
Ahstract This study aimed to compare the physical demands of contact small sided game (CSSG), non-contact small sided game (NCSSG) and match play in female rugby union. Fifteen female rugby union players participated within this study. Participants completed two testing sessions. Session one involved two 20-minute small sided games (contact and non-contact). Session two involved a competitive rugby union game. Movement characteristics, heart rate, PlayerLoad ${ }^{T M}$ and tackles were recorded using GPS units and heart rate monitors. No significant differences were identified between conditions in relation to average heart rate or time spent in different heart rate zones. Significant differences were identified between conditions for distance, normalised PlayerLoad ${ }^{\mathrm{TM}}$ and tackles. Distance covered was significantly higher in the non-contact small sided game, while normalised PlayerLoad ${ }^{\mathrm{TM}}$ and tackles were significantly lower in this condition. These findings suggest that the physiological stress, in terms of heart rate, is comparable between the match and both small sided games. Thus both small sided games seem to elicit an appropriate internal training response. However, the reductions in tackles and normalised PlayerLoad ${ }^{\top \mathrm{M}}$ in the non-contact small sided game are likely to reduce injury risk, while the increase in distance covered may enhance the training stimuli provided in this condition.
\end{abstract}

Key WOrds GPS, rugby, time motion analysis, small sided game, physical fitness

\section{Introduction}

Rugby Union is a physically demanding, invasion based intermittent team sport lasting over 80 minutes (Duthie, 2006). Throughout the course of match players engage in a number of high intensity activities such as sprinting, tackling, rucking, mauling, scrummaging and kicking interspersed with short periods of active and passive recovery (Bradley et al., 2015). The contact elements of a rugby union match-play frequently expose the players to high impact forces $(\sim 5,300 \mathrm{~N})$ (Seminati et al., 2017), thus increasing injury risk when compared to other team sports (Fuller, Sheerin, Targett, 2013). Contact accounts for the majority of rugby related injuries, with tackling 
the highest risk activity during both training and match play accounting for $44 \%$ and $45 \%$ of injuries to forwards and backs, respectively (Fuller et al., 2013).

Training is a vital part of rugby, with skills and fitness being developed during training sessions (Gabbett, Jenkins, Abernethy, 2012). It is essential that the physical preparation of players during training meets the specific requirements of match play. An understanding of the physical demands of match play is essential to informing the design of training interventions with a view to reducing injury risk and enhancing performance. Traditional conditioning, repeated high intensity effort exercise, and skills practice are all common methods of rugby training; however, all of these methods have been shown to elicit lower physical demands when compared to match play (Gabbett et al., 2012). Furthermore, traditional training methods commonly involve unopposed tasks, thus reducing ecological validity and limiting the opportunities for players to develop decision making skills in dynamic environments (Davids, Araújo, Correia, Vilar, 2013).

Small sided games (SSG) are becoming increasingly popular training tools within team sports as they provide a dynamic environment, that is easily modifiable, yet requires fewer players and reduced pitch sizes compared to the traditional game on which they are based (Hill-Hass, Dawson, Impellizzeri, Coutts, 2011). There is a growing body of evidence (Hill-Hass et al., 2011; Vaz, Goncalves, Figueira, Garcia, 2016; Impellizzeri et al., 2006; Gabbett, 2006) demonstrating the efficacy of SSG, due to comparable physiological demands between SSG and traditional conditioning activities or match play. Within rugby union, both contact SSG (CSSG) and non-contact SSG (NCSSG) are commonly used within training sessions (Fuller et al., 2013; Vaz et al., 2016; Jones, West, Crewther, Cook, Kilduff, 2015). However, to the author's knowledge, no studies have compared the physical demands of CSSG and NCSSG relative to each other or match play. If the physical demands of NCSSG match those of match play, this type of training may offer a means of maximising physiological adaptations while reducing injury risk by removing tackling. Previous work (Fuller et al., 2013) has demonstrated that non-contact skills based training has a lower injury rate (1.2/1,000 hours) compared to semi-contact (4.7/1,000 hours) and full contact skills $(6.1 / 1,000$ hours) based training.

Traditionally, rugby has been viewed as a male dominated sport but over recent years women's rugby union has grown in popularity (Chase, 2006). Despite this there is limited work exploring female rugby union. As such the aim of this study is to compare the physical demands of CSSG, NCSSG and match play, in female rugby union. Four hypotheses were tested within this study; (1) distance covered would be greater in the NCSSG; (2) time spent performing high speed movements would be greater in the NCSSG; (3) heart rate would be greater during the match and (4) PlayerLoad would be lower during the NCSSG.

\section{Methods}

\section{Participants}

Fifteen female rugby union players (19.8 \pm 1.6 years, $1.65 \pm 0.07 \mathrm{~m}, 70.5 \pm 13.1 \mathrm{~kg}$ ) participated in this study. All participants were recruited from a collegiate level Women's Rugby Team. Participants were free from injury for at least 6 months prior to testing and free from illness at the time of testing. All participants provided informed written consent and ethical approval was granted for this study by the Institutional Ethics Committee. 


\section{Procedures}

Participants attended two testing sessions. Both testing sessions were completed on an artificial $3 \mathrm{G}$ surface to reduce environmental changes to the surface between testing sessions (Burillo, Gallardo, Felipe, Gallardo, 2014). Participants completed standardised warm ups and cool downs at the start and end of each session. Session one required participants to complete two conditioned rugby specific SSG activities; NCSSG and CSSG. SSG were played on a $30 \mathrm{~m} \times 70 \mathrm{~m}$ standardised playing area (Johnston, Gabbett, Siebold, Jenkins, 2014). Pitch size and player numbers remained the same throughout both SSG. CSSG was played to standard 15 a side rugby union rules. NCSSG was played to touch rugby rules, with two handed taps replacing tackles. Once tackled, in the NCSSG, participants rolled the ball through their legs to a team mate, whilst the two players from the opposing team closest to the tackle area dropped to the floor and returned to their feet; thus creating space for the attacking side. After six tackles a turn over occurred. Both SSGs were played for 20 minutes with 20 minutes active recovery between each bout, reducing the effect of fatigue. The active recovery involved walking, light jogging and dynamic stretching. Session two was completed two weeks after session one. Within session two, participants competed in a BUCS Northern 1a league game played to full rugby union rules, for over 80 minutes. A schematic representation of the two testing sessions is presented in Figure 1.

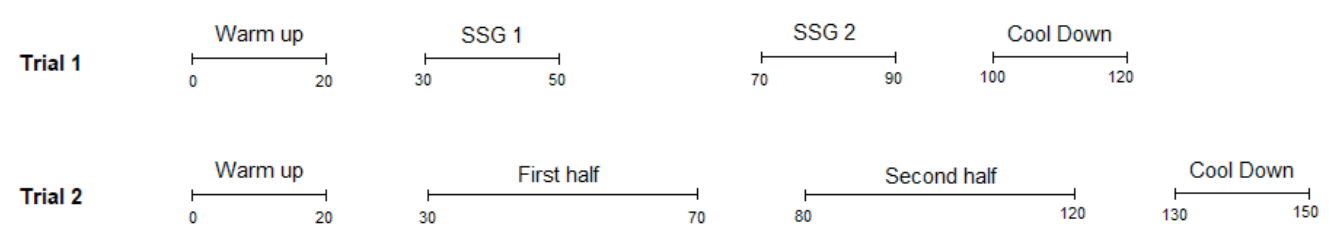

Figure 1. Schematic representation of the data collection sessions

Participants were required to continuously wear a Microelectromechanical systems (MEMS) device (MinimaxX, Catapult Sports, Melbourne, Australia). In an attempt to avoid erroneous data due to excessive movement artefact, the MEMS devices were housed between the participants scapulae in a standardised custom fitted neoprene garment worn directly against the participant's skin. Each MEMS device comprised a GPS component, 3D magnetometer and a tri-axial piezoelectric linear accelerometer (Kionix: KXP94) with sampling frequencies of 10, 30 and $100 \mathrm{~Hz}$ respectively. Each player wore the same device across matches to reduce any variation in GPS derived data due to potential between-unit discrepancies (Boyd, Ball, Aughey, 2011; Jennings, Cormack, Coutts, Boyd, Aughey, 2010). Acceptable inter-unit reliability has however been identified for the GPS [CV $=0.7-1.3 \%]$ and accelerometer [CV = 1.94\%] (Castellano, Casamichana, Calleja-Gonzalez, San Roman, Ostojic, 2011; Boyd et al., 2011) hardware contained within the MEMS devices used in the current study. Prior to the commencement of each season, all units were sent to the manufacturer for calibration using their preferred "jig" method. Units were orientated and secured in a stationary position in each plane of movement and recordings were set at $1 \mathrm{~g}$ for that position to reduce any bias or drift. Every two weeks, units were checked for calibration, with all units remaining within the manufacturer's tolerance thresholds during the entire testing period. In line with previous research (Malone, Lovell, Varley, Coutts, 
2017), GPS data was only included for statistical analyses if a horizontal dilution of precision (HDOP) of $<1.5$ and a number of satellites $\geq 6$ was achieved.

Data was downloaded from each GPS unit post-trial and analysed using Catapult Sprint Innovations 5.1.4 Team Sport Software (Catapult Innovations, Scoresby, Victoria). The following variables were extracted for analysis in each condition; average distance covered, time spent in working at different speeds, average HR, time spent in different HR zones, PlayerLoad ${ }^{T M}$ and tackles. Movement speed was classified into the following zones in accordance with M.R. Jones et al. (2015), low-speed movements: walking $\left(0-1.6 \mathrm{~m} \cdot \mathrm{s}^{-1}\right)$, jogging $\left(1.6-2.7 \mathrm{~m} \cdot \mathrm{s}^{-1}\right)$, cruising $\left(2.7-3.8 \mathrm{~m} \cdot \mathrm{s}^{-1}\right)$ and striding $\left(3.8-5.0 \mathrm{~m} \cdot \mathrm{s}^{-1}\right)$, and high-speed movement: high-intensity running (5.0$\left.5.5 \mathrm{~m} \cdot \mathrm{s}^{-1}\right)$ and sprinting $\left(>5.6 \mathrm{~m} \cdot \mathrm{s}^{-1}\right)$. Total playing time was determined for each player within the match and time off the field was removed from the data analysis. Prior to data analysis distance covered was converted to distance covered per minute $\left(\mathrm{m}^{\mathrm{min}} \mathrm{m}^{-1}\right)$ to enable direct comparisons between the three conditions, despite the differing durations. Average HR (b. $\mathrm{min}^{-1}$ ) and time spent in the following HR zones (Vaz et al., 2016) were extracted; < $75 \%$, $75-85 \%, 85-90 \%$ and $>90 \%$. PlayerLoad ${ }^{\top \mathrm{M}}$, the square root of the sum of the squared instantaneous rate of change acceleration in each of the three movement planes, was calculated from the accelerometer data (Catapult, 2013), within Catapult Sprint Innovations 5.1.4 Team Sport Software. PlayerLoad ${ }^{\mathrm{TM}}$ was normalised to both time and distance covered, to account for differences between the match and SSG durations and any potential changes in distance covered between conditions. Tackles were calculated from the accelerometer data recorded by the minimaxX unit, in accordance with manufacturer guidelines (Catapult, 2013) and normalised to time.

\section{Data Analysis}

All data is reported as mean $\pm S D$ unless otherwise stated. All statistical analysis was undertaken using IBM SPSS Statistics Editor 22 (IBM, Armonk, NY, USA). Prior to statistical analysis the assumption of normality was assessed using the Shapiro-Wilk test. All data were normally distributed. A repeated measures analysis of variance (ANOVA) was used to explore differences between conditions for distance, average HR, PlayerLoad ${ }^{\mathrm{TM}}$ and tackles. A $3 \times 4$ repeated measures ANOVA was used to explore the difference in the time spent within each HR zone across the three conditions and a $3 \times 6$ repeated measures ANOVA was used to explore for differences in time spent within each speed band between conditions. The sphericity assumption was checked using Mauchly's test of sphericity, where data violated the assumption the degrees of freedom were corrected using GreenhouseGeisser corrections. Pairwise comparisons were undertaken post-hoc where significant main effects were reported and the least significant difference correction was applied. The least significant difference was used to correct the level of significant for the pairwise comparisons, over the Bonferroni correction, as it is less conservative (Field, 2013). Partial eta squared $\left(\eta^{2}\right)$ was used as an estimate of effect size and interpreted as follows; small (0.01-0.059), moderate $(0.06-0.137)$ and large $(\geq 0.138)$. The level of significant was set at $p<0.05$.

\section{Results}

\section{Distance}

A significant main effect $\left(F=13.25, p=0.003, \eta^{2}=0.77\right)$ was reported for distance covered per minute (Figure 2A). Pairwise comparisons revealed a significant $(p<0.05)$ increase in distance covered during the NCSSG 
compared to both the match and CSSG. No significant difference $(p>0.05)$ was reported between the game and CSSG.
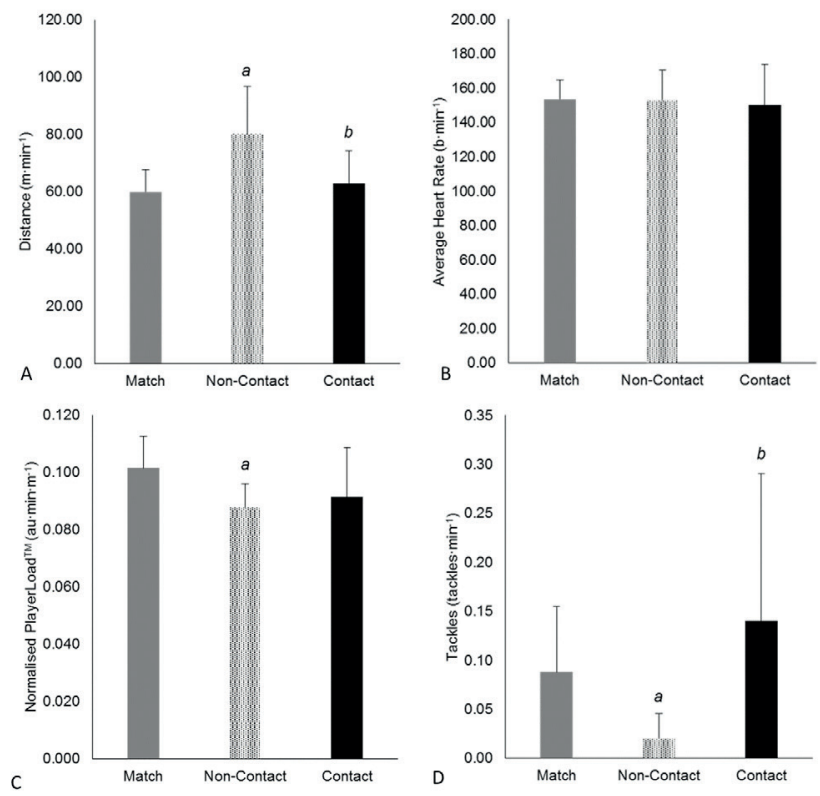

Figure 2. Results for the match (solid grey), non-contact small sided game (grey pattern) and contact small sided game (solid black). A. Distance covered per minute. B. Average heart rate. C. Normalised PlayerLoad ${ }^{\top M}$. D. Tackles. Data averaged across all participants. a denotes significantly different to the match. $b$ denotes significantly different to the NCSSG

\section{Speed}

Table 1 shows the percentage of time spent working in each speed zone during all conditions. On average participants spent the majority of time in each condition walking. More time was spent performing low speed movements in all conditions. A mixed design ANOVA revealed no significant condition*speed interaction $(F=2.51$, $\left.p=0.073, \eta^{2}=0.16\right)$.

Table 1. The percentage of time player's spent in each speed zone in all conditions

\begin{tabular}{lcccccc} 
& \multicolumn{3}{c}{ Low-speed Movements } & \multicolumn{2}{c}{ High-speed movements } \\
\cline { 2 - 7 } & walking (\%) & jogging (\%) & cruising (\%) & striding (\%) & HI running (\%) & sprinting (\%) \\
\hline Match & $53.6 \pm 11.0$ & $22.3 \pm 4.4$ & $15.8 \pm 5.8$ & $5.8 \pm 2.4$ & $1.1 \pm 0.8$ & $1.3 \pm 2.0$ \\
NCSSG & $49.4 \pm 7.80$ & $34.6 \pm 5.0$ & $12.2 \pm 4.3$ & $2.6 \pm 1.9$ & $0.2 \pm 0.4$ & $1.0 \pm 1.4$ \\
CSSG & $52.2 \pm 7.30$ & $28.4 \pm 7.7$ & $14.6 \pm 4.6$ & $3.2 \pm 2.9$ & $0.8 \pm 0.8$ & $0.8 \pm 1.3$ \\
\hline
\end{tabular}

NOTE: Values are averaged over all players. 


\section{Heart Rate}

Average $H R$ and the percentage of time spent working in each HR zone in each of the test conditions are shown in Figure $2 B$ and Table 2 respectively. No significant $\left(F=0.35, p=0.62, \eta^{2}=0.04\right.$ ) difference was reported for average heart rate between conditions. Participants spent more time working at $<75 \% \mathrm{HRmax}$ and $75-85 \% \mathrm{HRmax}$ in all conditions. No significant $\left(F=0.36, p=0.77, \eta^{2}=0.02\right)$ condition*zone interactions were reported.

Table 2. The percentage of time players spent in each heart rate zone and average heart rate during each condition

\begin{tabular}{lcccc}
\cline { 2 - 4 } & \multicolumn{4}{c}{ Heart Rate $(\%)$} \\
\cline { 2 - 4 } & $<75$ & $75-85$ & $85-90$ & $<90$ \\
\hline Match & $37.9 \pm 24.3$ & $38.6 \pm 13.6$ & $14.9 \pm 8.7$ & $8.6 \pm 7.4$ \\
NCSSG & $41.6 \pm 34.7$ & $29.6 \pm 17.9$ & $14.5 \pm 11.6$ & $14.3 \pm 21.0$ \\
CSSG & $34.9 \pm 33.2$ & $37.5 \pm 19.7$ & $18.5 \pm 15.3$ & $9.1 \pm 10.9$ \\
\hline
\end{tabular}

NOTE: Values are averaged over all players.

\section{Normalised PlayerLoadi'}

A significant main effect $\left(F=6.39, p=0.02, \eta^{2}=0.62\right)$ was reported for normalised PlayerLoad ${ }^{\mathrm{TM}}$ (Figure $2 \mathrm{C}$ ). Pairwise comparisons revealed that normalised PlayerLoad ${ }^{\mathrm{TM}}$ was significantly $(p=0.02)$ lower during the NCSSG compared to the match. No significant $(p>0.05)$ differences were reported between the CSSG and either the match or NCSSG.

\section{Tackles}

A significant main effect $\left(F=4.17, p=0.04, \eta^{2}=0.23\right)$ was reported for tackles per minute (Figure 2D). Pairwise comparisons revealed that significantly $(p<0.05)$ fewer tackles were made per minute of play in the NCSSG compared to both the match and CSSG. No significant $(p=0.92)$ difference was reported between the match and CSSG for tackles per minute.

\section{Discussion}

To the authors knowledge this is the first study to explore the physical demands of CSSG and NCSSG relative to match play, despite contact and non-contact SSG commonly being used during rugby union training sessions (Vaz et al., 2016; Jones et al., 2015). The findings of this study support two of the four proposed hypotheses, with greater distance covered and lower PlayerLoad ${ }^{\mathrm{TM}}$ during the NCSSG compared to the CSSG and match play. These findings suggest that there are important differences in a number of key metrics that may influence injury risk and training stimuli. It was also identified that no significant differences existed for the heart rate data or time spent within each speed zone between the NCSSG, CSSG and match data. These data therefore suggest that the relative intensity of the two training sessions was not significantly different to that of match play; these findings do however reject hypotheses two and three.

The distance covered per minute was significantly increased during the NCSSG compared to both the match and the CSSG (Figure 2). This increase in distance covered per minute within the NCSSG highlights an increase in average speed during this condition. The match and the CSSG have highly comparable distances covered and 
average speeds suggesting that the CSSG replicates the demands of the match. In relation to training the increases in distance covered per minute during the NCSSG would equate to covering an extra $516 \mathrm{~m}$ over the course of a 30 minute training session in comparison to the CSSG condition. This substantial increase in distance covered between the conditions would therefore suggest the NCSSG would elicit the greatest training stimuli. Increasing the training stimuli in this way would likely increase the positive adaptations to the aerobic system, which in turn may help players maintain higher work rates within match play. Previous studies (Castagna, Manzi, Impellizzeri, Weston, Barbero Alvarez, 2010; laia, Rampinini, Bangsbo, 2009) have demonstrated that teams who cover more distance during match play and who are aerobically fitter have higher performance levels.

Time motion analysis was undertaken to further explore the changes in movement characteristics between the three conditions (Table 1). This analysis revealed that participants spent more time jogging and less time walking during the NCSSG compared to both the match and CSSG. These changes in time spent walking and jogging between conditions may explain the changes in distance covered. However, it must be noted that no significant condition*speed interaction was reported within this study and that, in all conditions, high-speed movements were limited, accounting for $\leq 2 \%$ of the total time (Table 1).

Despite the significant changes in distance covered per minute between conditions there were no significant differences in average HR (Table 2). The HR data therefore suggests comparable internal workloads across the three test conditions, with both SSG replicating the demands of the match. As such, either type of SSG could be used to prepare players for match play. The lack of any significant changes in HR between the three conditions is surprising given the significant increases in distance covered and thus average speed in the NCSSG. A possible reason for the similar HR responses to each condition may be the contact element of the match and CSSG. The contact element adds a resistive factor that may help to elevate the HR during the match and CSSG (Deustch, Maw, Jenkins, Reaburn, 1998; Bowen, Gross, Gimpel, Li, 2017). While the contact element of the CSSG may help to explain the HR findings, it may also increase the risk of injury, as the majority of rugby related injuries are a result of contact (Mclntosh, Savage, McRory, Fréchède, Wolfe, 2010; Fuller, Sheerin, Targett, 2013). Removing the contact element from training may therefore help to reduce the risk of injury.

Removing the tackling aspect of the game in the NCSSG resulted in significant reductions in both the tackles per minute and normalised PlayerLoad ${ }^{\mathrm{TM}}$ (Figure $2 \mathrm{C}$ and $\mathrm{D}$ ) metrics. The significant reduction in tackles in the NCSSG compared to the CSSG and match is unsurprising given the rules associated with this conditioned SSG. The reduction in tackles during the NCSSG may explain the reduction in normalised PlayerLoad ${ }^{\mathrm{TM}}$. PlayerLoad ${ }^{\mathrm{TM}}$ has previously been linked to injury risk (Bowen et al., 2017), therefore the significant reduction in both this metric and tackles supports the suggestion that injury risk should be reduced in the NCSSG compared to both the CSSG and match play. In contrast, the match and NCSSG are more closely matched in terms of normalised PlayerLoad ${ }^{\mathrm{TM}}$ and tackles per minute. There was a non-significant increase in tackles per minute during the CSSG compared to the match, however this was not replicated in the normalised PlayerLoad ${ }^{\mathrm{TM}}$ data, which was higher in the match than the CSSG. This slight reduction in PlayerLoad ${ }^{T M}$ in the CSSG compared to the match, despite the increase in tackles per minute suggests that the tackles made in the CSSG are of a lower intensity. Thus the injury risk in the CSSG may still be lower than in the match.

The findings of this study need to be interpreted in light of a number of factors. This study was completed on artificial $3 G$ turf. Artificial $3 G$ turf was used within this study to reduce environmental changes to the surface between testing sessions (Burillo et al., 2014). While the use of artificial surfaces is increasing within rugby union 
(Cunniffe, Proctor, Baker, 2009), studies have demonstrated differences in ground reaction forces and running velocities compared to natural turf (Stiles, James, Dixon, Guisasola, 2009; McGhie, Ettema, 2013). For this reason future work should replicate the current study on natural turf. Finally, the specific design of the SSG undertaken within this study may have altered the manner in which players acted within each session. Both the CSSG and NCSSG were standardised by encouraging players to play with width. For this reason future work exploring the influence of contact and non-contact SSG with different objectives may be warranted.

\section{Conclusions}

In conclusion, this study provides a novel insight into the physical demands of common training methods and match play in female rugby union players. The findings of this study revealed that both NCSSG and CSSG appear to replicate the physical demands of match play. As such either type of SSG appear to offer appropriate training methods for female rugby union players. The NCSSG may offer an enhanced training stimuli though, due to the increased distance covered per minute compared to the CSSG. In addition, the NCSSG is also liable to reduce injury risk in comparison to the CSSG as the majority of rugby related injuries are a direct result of contact.

\section{References}

Bowen, L., Gross, A.S., Gimpel, M., Li, F.X. (2017). Accumulated workloads and the acute: chronic workload ration relate to injury risk in elite youth football players. British Journal of Sports Medicine, 51 (5), 452-459. DOI: 10.1136/bjsports-2015-095820.

Boyd, L.J., Ball, K., Aughey, R.J. (2011). The reliability of minimaxX accelerometers for measuring physical activity in Australian football. International Journal of Sports Physiology and Performance, 6 (3), 311-321.

Bradley, W.J., Cavanagh, B.P., Douglas, W., Donovan, T.F., Morton, J., Close, G.L. (2015). Quantification of training load, energy intake and physiological adaptations during a rugby preseason: a case study form an elite rugby union squad. Journal of Strength and Conditioning Research, 29 (2), 534-544. DOI: 10.1519/JSC.0000000000000631.

Burillo, P., Gallardo, L., Felipe, J.L., Gallardo, A.M. (2014). Artificial turf surfaces: perception of safety, sporting feature, satisfaction and preference of football users. European Journal of Sport Science, 14 (S1), 437-447. DOI: 10.1080/17461391.2012.713005.

Castagna, C., Manzi, V., Impellizzeri, F., Weston, M., Barbero Alvarez, J.C. (2010). Relationship between endurance field tests and match performance in young soccer players. Journal of Strength and Conditioning Research, 24 (12), 3227-3233. DOI: 10.1519/ JSC.0b013e3181e72709.

Castellano, J., Casamichana, D., Calleja-Gonzalez, J., San Roman, J., Ostojic, S.M. (2011). Reliability and accuracy of 10 Hz GPS devices for short-distance exercise. Journal of Sports Science and Medicine, 10, 233-234.

Catapult (2013). Sprint Help Manual.

Chase, L.F. (2006). (Un)Disciplined bodies: A foucauldian analysis of women's rugby. Sociology of Sport Journal, 23 (3), $229-247$. DOI: 10.1123/ssj.23.3.229.

Cunniffe, B., Proctor, W., Baker, J.S., Davies, B. (2009). An evaluation of the physiological demands of elite rugby union using global positioning system tracking software. Journal of Strength and Conditioning Research, 23 (4), 1195-2003.

Davids, K., Araújo, D., Correia, V., Vilar, L. (2013). How small-sided games and conditioned games enhance acquisition of movement and decision making skills. Exercise and Sport Science Reviews, 41 (3), 154-161.

Deustch, M.U., Maw, G.J., Jenkins, D., Reaburn, P. (1998). Heart rate, blood lactate and kinematic data of elite colts (under-19) rugby union players during competition. Journal of Sports Sciences, 16 (6), 5561-5570.

Duthie, G.M. (2006). A framework for the physical development of elite rugby union players. International Journal of Sports Physiology and Performance, 1 (1), 2-13.

Field, A. (2013). Discovering Statistics using IBMM SPSS Statistics $4^{\text {th }}$ Ed. London: Sage.

Fuller, C.W., Sheerin, K., Targett, S. (2013). Rugby World Cup 2011: International Rugby Board Injury Surveillance Study. British Journal of Sports Medicine, 47, 1184-1191. 
Gabbett, T.J. (2006). Skill-based conditioning games as an alternative to traditional conditioning for rugby league players. Journal of Strength and Conditioning Research, 20 (2), 309-315.

Gabbett, T.J., Jenkins, D.J., Abernethy, B. (2012). Physical demands of professional rugby league training and competition using microtechnology. Journal of Science and Medicine in Sport, 15 (1), 80-86. DOI: 10.1016/j.jsams.2011.07.004.

Hill-Haas, S.V., Dawson, B., Impellizzeri, F.M., Coutts, A.J. (2011). Physiology of small-sided games training in football: a systematic review. Sports Medicine, 41 (3), 199-220. DOI: 10.2165/11539740-000000000-00000.

laia, F.M., Rampinini, E., Bangsbo, J. (2009). High intensity training in football. International Journal of Sports Physiology and Performance, 4 (3), 291-306.

Impellizzeri, F.M., Marcora, S.M., Castagna, C., Reilly, T., Sassi, A., laia, F.M., Rampinini, E. (2006). Physiological and performance effects of generic versus specific aerobic training in soccer players. International Journal of Sports Medicine, 27 (6), 483-492.

Jennings, D., Cormack, S., Coutts, A.J., Boyd, L., Aughey, R.J. (2010). The validity and reliability of GPS units for measuring distance in team sport specific running patterns. International Journal of Sports Physiology and Performance, 5 (3), 328-341.

Johnston, R.D., Gabbett, T.J., Siebold, A.J., Jenkins D.G. (2014). Influence of physical contact on neuromuscular fatigue and markers of muscle damage following small sided games. Journal of Science and Medicine in Sport, 17 (5), 535-540. DOI: 10.1016/j. jsams.2013.07.018.

Jones, M.R., West, D.J., Crewther, B.T., Cook, C.J., Kilduff, L.P. (2015). Quantifying positional and temporal movement patterns in professional rugby union using global positioning system. European Journal of Sport Science, 15 (6), 488-496. DOI: 10.1080/17461391.2015.1010106.

Malone, J.J., Lovell, R., Varley, M.C., Coutts, A.J. (2017). Unpacking the black box: applications and considerations for using GPS devices in sport. International Journal of Sports Physiology and Performance, 12 (S2), 218-226.

McGhie, D., Ettema, G. (2013). Biomechanical analysis of surface-athlete impacts on third-generation artificial turf. The American Journal of Sports Medicine, 41 (1), 177-185. DOI: 10.1177/0363546512464697.

McIntosh, A.S., Savage, T.N., McRory, P., Fréchède, B.O., Wolfe, R. (2010). Tackle characteristics and injury in a cross section of rugby union football. Medicine and Science in Sports and Exercise, 42 (5), 977-984.

Seminati, E., Cazzola, D., Preatoni, E., Stokes, K., Williams, S., Trewartha G. (2017). Tackle direction and dominant side effect upper body loading during rugby tackles. British Journal of Sports Medicine, 51, 386. DOI: 10.1136/bjsports-2016-097372.259.

Stiles, V.H., James, I.T., Dixon, S.J., Guisasola, I.N. (2009). Natural turf surfaces: the case for continued research. Sports Medicine, 39 (1), 65-84. DOI: 10.2165/00007256-200939010-00005.

Vaz, L.M.T., Goncalves, B.S.V., Figueira, B.E.N., Garcia, G.C. (2016). Influence of different small-sided games on physical and physiological demands in rugby union players. International Journal of Sports Science and Coaching, 11, 78-84. DOl: 10.1177/1747954115624823.

Cite this article aS:" Chadwick, L., Page, R.M., Langley, B. (2019). Quantifying the Physical Demands of Small Sided Games in Rugby Union: Contact vs. Non-contact. Central European Journal of Sport Sciences and Medicine, 2 (26), 5-13. DOI: 10.18276/ cej.2019.2-01. 